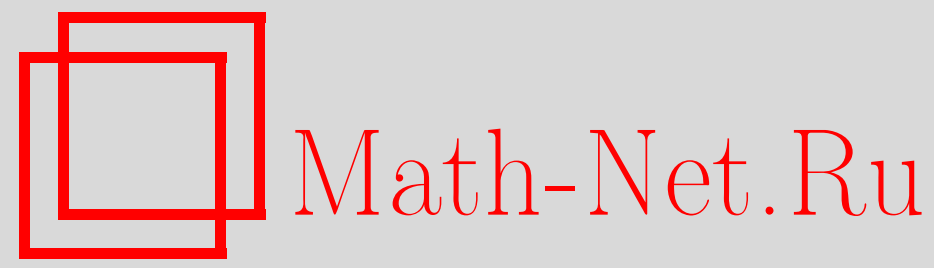

В. А. Копытцев, В. Г. Михайлов, О распределении чисел решений случайных включений, Матем. вопр. криптогр., 2011, том 2, выпуск 2, 55-80

DOI: https://doi.org/10.4213/mvk31

Использование Общероссийского математического портала Math-Net.Ru подразумевает, что вы прочитали и согласны с пользовательским соглашением

http://www.mathnet.ru/rus/agreement

Параметры загрузки:

IP : 3.82 .47 .9

26 апреля 2023 г., 17:56:23 
УДК: 519.212.2

\title{
О распределении чисел решений случайных включений
}

\author{
В. А. Копытцев ${ }^{1}$, В. Г. Михайлов ${ }^{2}$ \\ ${ }^{1}$ Академия криптографии Российской Федерации, Москва \\ ${ }^{2}$ Математический институт им. В. А. Стеклова РАН, Москва
}

Получено 25.I.2011

При заданных множествах $D$ и $B$ векторов линейных пространств $V^{n}$ и $V^{T}$ над полем $K=G F(q)$ изучается распределение числа решений $\xi(D, F, B)$ системы включений $x \in D, A_{1} x+A_{2} f(x) \in B$, где $A_{1}$ и $A_{2}-$ случайные матрицы над полем $K$ размеров $T \times n$ и $T \times m$ с независимыми элементами, а $f: V^{n} \rightarrow V^{m}$ заданное отображение. Указаны условия сходимости распределений случайных величин $\xi(D, F, B)$ к простому или сложному распределениям Пуассона. Результаты применяются к числу решений системы случайных полиномиальных уравнений.

Ключевые слова: случайные включения, системы случайных уравнений, число решений, предельная теорема Пуассона

On the distribution of the numbers of solutions of random inclusions

\author{
V. A. Kopytcev' ${ }^{1}$, V. G. Mikhailov ${ }^{2}$ \\ ${ }^{1}$ Academy of Cryptography of Russian Federation, Moscow \\ ${ }^{2}$ Steklov Mathematical Institute of RAS, Moscow
}

Abstract. For given sets $D$ and $B$ of vectors in linear spaces $V^{n}$ and $V^{T}$ over the field $K=G F(q)$ we consider the number of solutions $\xi(D, F, B)$ of the system of inclusions $x \in D, A_{1} x+A_{2} f(x) \in B$, where $A_{1}$ and $A_{2}$ are random $T \times n$ and $T \times m$ matrices over $K$ with independent elements and $f: V^{n} \rightarrow V^{m}$ is a given mapping. Sufficient conditions for the convergence of distributions of $\xi(D, F, B)$ to the Poisson or compound Poisson distributions are found. Results are applied to the number of solutions of a system of random polynomial equations.

Key words: random inclusions, systems of random equations, number of solutions, Poisson limit theorem

Citation: Mathematical Aspects of Cryptography, 2011, vol. 2, no. 2, pp. 55-80 (Russian). 


\section{1. Введение}

В работе исследуется распределение числа решений $\xi(D, F, B)$ системы включений

$$
x \in D, \quad F(x) \in B,
$$

где $F(x)=\left(F_{1}(x), \ldots, F_{T}(x)\right): V^{n} \rightarrow V^{T}-$ случайное отображение пространства $n$-мерных векторов $V^{n}$ над полем $K=G F(q)$ в пространство $V^{T}$, а $D$ и $B$ - некоторые множества $n$-мерных и $T$-мерных векторов над этим полем. В случае, когда $D=V^{n}$, а множество $B$ состоит из единственного вектора $B=\left\{b=\left(b_{1}, \ldots, b_{T}\right)\right\}$, включения (1.1) эквивалентны системе из $T$ случайных уравнений $F_{j}(x)=b_{j}, \quad j=1, \ldots T$, относительно векторов $x \in V^{n}$.

Вместе с распределением величины $\xi(D, F, B)$ исследуется распределение числа $\xi\left(D, F, B \mid x^{0}\right)$, равного количеству решений системы включений

$$
x \in D, \quad F(x) \in F\left(x^{0}\right)+B,
$$

где $F\left(x^{0}\right)+B=\left\{F\left(x^{0}\right)+b \mid b \in B\right\}$, а $x^{0} \in V^{n}$ - заданный вектор. В случае, когда $D=V^{n}$ и $B=\left\{0^{T}\right\}$, система включений (1.2) обращается в «заведомо совместную» систему уравнений $F_{j}(x)=F_{j}\left(x^{0}\right), \quad j=1, \ldots T$, относительно векторов $x \in V^{n}$.

Поскольку заменой переменных $u=x-x^{0}$ включения (1.2) приводятся к виду (1.1)

$$
u \in D-x^{0}, \quad F^{\left(x^{0}\right)}(u) \in B,
$$

где $F^{\left(x^{0}\right)}(u)=F\left(u+x^{0}\right)-F\left(x^{0}\right)$, между величинами $\xi\left(D, F, B \mid x^{0}\right)$ и величинами $\xi(D, F, B)$ существует связь, выраженная равенством

$$
\xi\left(D, F, B \mid x^{0}\right)=\xi\left(D-x^{0}, F^{\left(x^{0}\right)}, B\right) .
$$

При этом $\xi\left(D, F, B \mid 0^{n}\right)=\xi(D, F, B)$, если $F\left(0^{n}\right)=0^{T}$.

Рассмотрим специальный класс включений (1.1). Пусть $F(x)=A_{1} x+$ $+A_{2} f(x)$ при случайных матрицах $A_{1}$ и $A_{2}$ над полем $K=G F(q)$ размерностей $T \times n$ и $T \times m$ соответственно с независимыми в совокупности случайными элементами и при заданном отображении $f(x)=\left(f_{1}(x), \ldots, f_{m}(x)\right)$ : $V^{n} \rightarrow V^{m}$, удовлетворяющем условию $f\left(0^{n}\right)=0^{m}$. Класс включений с таким отображением $F$ представляет особый интерес, поскольку в него входят системы полиномиальных уравнений

$$
\sum_{\substack{d_{1}, \ldots, d_{n} \in\{0, \ldots, q-1\} \\ 1 \leq d_{1}+\ldots+d_{n} \leq g}} a_{d_{1} \ldots d_{n}}^{(t)} x_{1}^{d_{1}} \ldots x_{n}^{d_{n}}=b_{t}, \quad t=1, \ldots, T .
$$

\section{МАТЕМАТИЧЕСКИЕ ВОПРОСЫ КРИПТОГРАФИИ}


В случае, когда $F(x)=A_{1} x+A_{2} f(x)$, число решений системы включений (1.1) совпадает с числом решений системы линейных включений

$$
y \in D_{f}, \quad A y \in B
$$

где

$$
y=(x, f(x)), \quad D_{f}=\{(x, f(x)) \mid x \in D\} \subseteq V^{n+m},
$$

а матрица $A=\left(A_{1}, A_{2}\right)$ получена объединением столбцов матриц $A_{1}, A_{2}$. Значит,

$$
\xi(D, F, B)=\xi\left(D_{f}, A, B\right) .
$$

Равенства (1.5) и (1.3) позволяют использовать для изучения распределений случайных величин $\xi(D, F, B)$ и $\xi\left(D, F, B \mid x^{0}\right)$ результаты работы [1].

В работе [1] был изучен случай, когда $f(x) \equiv 0^{m}, F(x)=A_{1} x$. Были выведены условия сходимости при $n, T \rightarrow \infty$ распределения числа решений системы включений $x \in D, A_{1} x \in B$ к распределениям пуассоновского типа. Решения включения $A_{1} x \in B$ (или системы линейных уравнений $A_{1} x=b$ ), удовлетворяющие условию $x \in D, D \subset V^{n}$, принято называть специальными. В работах [3], [4] в качестве множеств $D$ рассматривались шары и сферы относительно метрики Хемминга в пространстве $V^{n}$. В работе [7] была описана связь между числом специальных решений случайных однородных линейных уравнений $A_{1} x=0$ и числом решений специально построенного случайного линейного включения $A^{\prime} y \in B^{\prime}$. Итог этим исследованиям был подведен в работе [1].

Исследование распределений величин $\xi(D, F, B)$ с использованием равенств (1.5) впервые проведено в работе [2]. Главная особенность работы [2] состоит в том, что в ней ослаблены асимптотические условия для распределений элементов матрицы $A$ по сравнению с условиями из работы [1].

Сформулируем результаты настоящей работы. Далее предполагаем, что от параметров $n, T$ зависят числа $m=m(n)$, множества $D=D(n), B=B(T)$ и матрицы $A=A(n, T)$.

Обозначим через $N\left(a_{1}, a_{2}, a_{3}, d, B\right)$ число решений уравнения $a_{1} u^{1}+$ $+a_{2} u^{2}+a_{3} u^{3}=d$ относительно тройки векторов $\left(u^{1}, u^{2}, u^{3}\right) \in B^{3}$, где $a_{1}, a_{2}, a_{3} \in K \backslash\{0\}, d \in V^{T}$. Пусть

$$
\begin{gathered}
N(B)=\max _{a_{1}, a_{2}, a_{3}, d} N\left(a_{1}, a_{2}, a_{3}, d, B\right), \\
\rho(B)=N(B) /|B|^{2} .
\end{gathered}
$$


Очевидно, что $0 \leq \rho(B) \leq 1$. Если $B$ - линейное или аффинное подпространство, то эта величина принимает максимально возможное значение $\rho(B)=1$ (см. [7]).

Ниже будут играть важную роль условие $\rho(B(T)) \rightarrow 0$ при $T \rightarrow \infty$ и условие $\rho(D(n)) \rightarrow 0$ при $n \rightarrow \infty$. Пусть $\|b\|$ означает количество ненулевых координат вектора $b \in V^{T}$. Согласно теореме 1 [1] условие $\rho(B(T)) \rightarrow 0$ при $T \rightarrow \infty$ выполнено, в частности, для множеств

$$
\begin{gathered}
S_{r}^{T}\left(b^{*}\right)=\left\{b \in V^{T}: 1 \leq\left\|b-b^{*}\right\| \leq r\right\}, \\
\hat{S}_{r}^{T}\left(b^{*}\right)=\left\{b \in V^{T}:\left\|b-b^{*}\right\|=r\right\}
\end{gathered}
$$

при любых векторах $b^{*} \in V^{T}$, если $1 \leq r$ и $r T^{-1} \leq \varepsilon$, где $0<\varepsilon<(q-1) / q-$ заданное число, а $q$ - число элементов поля $K=G F(q)$. Аналогичное утверждение справедливо для величины $\rho(D(n))$.

Пусть элементы случайной матрицы $A=\left(A_{1}, A_{2}\right)$ независимы в совокупности и распределены с вероятностями

$$
\boldsymbol{P}\left\{a_{i, j}=k\right\}=\frac{1+\Delta_{i, j}(k)}{q}, \quad k \in K,
$$

где $\sum_{k \in K} \Delta_{i, j}(k)=0, i=1, \ldots, T, j=1, \ldots, n+m$.

Пусть

$$
\Delta=\max _{i, j, k}\left|\Delta_{i, j}(k)\right|<1 .
$$

Элементы $(x, b)$ и $\left(x^{\prime}, b^{\prime}\right)$ множества $D \times B$ назовем подобными, если $\left(x^{\prime}, b^{\prime}\right)=$ $=(k x, k b)$ при некотором элементе $k \in K \backslash\{0\}$. Разобьем множество $D \times B$ на классы $(D B)_{1}, \ldots,(D B)_{M}$ подобных векторов, где $M-$ общее число таких классов. Положим

$$
l_{r}(D, B)=\left|\left\{u \in\{1, \ldots, M\}:\left|(D B)_{u}\right|=r\right\}\right|, \quad r=1, \ldots, q-1 .
$$

Пусть $\pi_{1}\left(\lambda_{1}\right), \pi_{2}\left(\lambda_{2}\right), \ldots, \pi_{q-1}\left(\lambda_{q-1}\right)$ - независимые в совокупности случайные величины, распределенные по закону Пуассона с параметрами $\lambda_{1}, \lambda_{2}, \ldots, \lambda_{q-1}$ соответственно.

Теорема 1. Пусть $D \subseteq V^{n} \backslash\left\{0^{n}\right\}$, выполнены условия $n, T \rightarrow \infty, T \Delta \rightarrow$ $\rightarrow 0,|D| \rightarrow \infty$ и соотношения

$$
\begin{gathered}
\rho\left(D_{f}\right) \rho(B) \rightarrow 0, \\
q^{-T} l_{r}\left(D_{f}, B\right) \rightarrow \lambda_{r}, \quad 0 \leq \lambda_{r}<\infty, \quad r=1, \ldots, q-1, \\
\exists r \in\{1, \ldots, q-1\}: \lambda_{r}>0 .
\end{gathered}
$$


Тогда распределение случайной величины $\xi(D, F, B)$ сходится к распределению случайной величинь

$$
\pi\left(\lambda_{1}, \ldots, \lambda_{q-1}\right)=\pi_{1}\left(\lambda_{1}\right)+2 \pi_{2}\left(\lambda_{2}\right)+\ldots+(q-1) \pi_{q-1}\left(\lambda_{q-1}\right) .
$$

Определим отображение $f^{\left(x^{0}\right)}: V^{n} \rightarrow V^{m}$ равенствами $f^{\left(x^{0}\right)}(x)=f(x+$ $\left.+x^{0}\right)-f\left(x^{0}\right)$. Будем предполагать, что вектор $x^{0}=x^{0}(n)$ зависит от параметра $n$.

Теорема 1а. Пусть $D \subseteq V^{n} \backslash\left\{x^{0}\right\}$, выполнены условия $n, T \rightarrow \infty$, $T \Delta \rightarrow 0,|D| \rightarrow \infty$ и соотношения

$$
\begin{gathered}
\rho\left(\left(D-x^{0}\right)_{f^{\left(x^{0}\right)}}\right) \rho(B) \rightarrow 0, \\
q^{-T} l_{r}\left(\left(D-x^{0}\right)_{f^{\left(x^{0}\right)}}, B\right) \rightarrow \lambda_{r}, \quad 0 \leq \lambda_{r}<\infty, \quad r=1, \ldots, q-1, \\
\exists r \in\{1, \ldots, q-1\}: \lambda_{r}>0 .
\end{gathered}
$$

Тогда распределение случайной величины $\xi\left(D, F, B \mid x^{0}\right)$ сходится к распределению случайной величины $\pi\left(\lambda_{1}, \ldots, \lambda_{q-1}\right)$.

ЗАМЕчАНИЕ 1. Теорема 1 вытекает непосредственно из теоремы 3 работы [1] и равенств (1.5). Теорема 1а следует из теоремы 1, равенств (1.3) и $F^{\left(x^{0}\right)}(x)=F\left(x+x^{0}\right)-F\left(x^{0}\right)=A_{1} x+A_{2} f^{\left(x^{0}\right)}(x)$. Из условия $f\left(0^{n}\right)=0^{m}$ следует, что $f^{\left(0^{n}\right)}(x)=f(x)$. Поэтому теорема 1а обратится в теорему 1 , если положить $x^{0}=0^{n}$.

ЗАМЕчАНИЕ 2. Из результатов работы [2] следует, что в некоторых случаях условие $T \Delta \rightarrow 0$, использованное в теоремах 1 и 1а, можно ослабить. Так, из теоремы 4 работы [2] и равенств (1.3) вытекает, что справедливо утверждение, получающееся из теоремы 1а после замены условий $D \subseteq V^{n} \backslash\left\{x^{0}\right\}$ и $T \Delta \rightarrow 0$ условиями $D=V^{n} \backslash\left\{x^{0}\right\}$ и $\Delta \rightarrow 0$ соответственно, и включения в перечень условий соотношения $|B| \leq q^{\delta T}$, где $0 \leq \delta<1-$ заданное число.

ЗАМЕЧАНИЕ 3. Согласно определениям

$$
\rho\left(\left(D-x^{0}\right)_{f^{\left(x^{0}\right)}}\right) \leq \rho\left(D-x^{0}\right)=\rho(D) .
$$

Поэтому соотношение $\rho(D) \rightarrow 0$ влечет выполнение условия (1.11). 


$$
\begin{aligned}
& \text { Пусть } D=V^{n} \backslash\left\{x^{0}\right\}, \text { тогда } D-x^{0}=V^{n} \backslash\left\{0^{n}\right\} \text { и } \\
& \rho\left(\left(D-x^{0}\right)_{f_{\left(x^{0}\right)}}\right)=\rho\left(\left(V^{n} \backslash\left\{0^{n}\right\}\right)_{f\left(x^{0}\right)}\right) .
\end{aligned}
$$

Приведем условия, влекущие соотношение

$$
\rho\left(\left(V^{n} \backslash\left\{0^{n}\right\}\right)_{f_{\left(x^{0}\right)}}\right) \rightarrow 0
$$

и, следовательно, выполнение условия (1.11).

Положим

$$
R(f)=\left\{f_{1}(x), \ldots, f_{m}(x)\right\} .
$$

Введем при $l=2,3, \ldots$ множество функций

$$
Q\left(d^{1}, \ldots, d^{l}\right)=\bigcup_{s=1}^{l}\left\{x_{1}^{d_{1}^{s}} \ldots x_{n}^{d_{n}^{s}}\right\}
$$

где $d^{s}=\left(d_{1}^{s}, \ldots, d_{n}^{s}\right) \in\{0,1\}^{n}, s=1, \ldots, l$, при этом

$$
\begin{gathered}
\left\|d^{s}\right\| \geq 2, \quad s=1, \ldots, l, \\
\left\|\left(d_{1}^{s_{1}} d_{1}^{s_{2}}, \ldots, d_{n}^{s_{1}} d_{n}^{s_{2}}\right)\right\|=0, \quad 1 \leq s_{1}<s_{2} \leq l .
\end{gathered}
$$

Согласно условию (1.15) множества существенных переменных функций $x_{1}^{d_{1}^{s}} \ldots x_{n}^{d_{n}^{s}}$ из набора $Q\left(d^{1}, \ldots, d^{l}\right)$ попарно не пересекаются, а условие (1.14) означает, что степень нелинейности функций $x_{1}^{d_{1}^{s}} \ldots x_{n}^{d_{n}^{s}}$ больше единицы.

Будем предполагать, что векторы $d^{s}=d^{s}(n), s=1, \ldots, l$, и величина $l=l(n)$ зависят от параметра $n$.

Теорема 2. Пусть $F(x)=A_{1} x+A_{2} f(x), R(f) \supseteq Q\left(d^{1}, \ldots, d^{l}\right), l \leq m$, $n \rightarrow \infty, u$

$$
\prod_{s=1}^{l}\left(1-q^{-2\left\|d^{s}\right\|}\right) \rightarrow 0 .
$$

Тогда выполнено соотношение

$$
\rho\left(\left(V^{n} \backslash\left\{0^{n}\right\}\right)_{f^{\left(x^{0}\right)}}\right) \rightarrow 0,
$$

равномерно относительно векторов $x^{0} \in V^{n}$.

Скажем, что для множества $B$ выполнено условие (В), если $B$ разбивается на тривиальные классы подобных векторов, содержащие по одному элементу. Условие (В), например, выполнено для множеств $B=\{b=$ $\left.=\left(b_{1}, \ldots, b_{T}\right)\right\}$, состоящих из единственного вектора, а в случае $K=G F(2)$ для всех множеств $B \subseteq V^{T}$.

Непосредственно из теорем 1a, 2 вытекает следствие 1. 
Следствие 1. Пусть $F(x)=A_{1} x+A_{2} f(x), R(f) \supseteq Q\left(d^{1}, \ldots, d^{l}\right)$, выполнены условия (B), $n, T \rightarrow \infty, T \Delta \rightarrow 0$, (1.16) и соотночение

$$
q^{n-T}|B| \rightarrow \lambda, \quad 0<\lambda<\infty .
$$

Тогда распределения случайных величин $\xi\left(V^{n} \backslash\left\{x^{0}\right\}, F, B \mid x^{0}\right)$ (и в том числе распределение величины $\left.\xi\left(V^{n} \backslash\left\{0^{n}\right\}, F, B\right)\right)$ сходятся $\kappa$ распределению Пуассона с параметром $\lambda$ равномерно относительно векторов $x^{0} \in V^{n}$.

ЗАМЕчАниЕ 4. Условия теоремы 2 выполнены для $R(f) \supseteq Q_{n}^{(t)}$, где $t \geq 2$,

$$
Q_{n}^{(t)}=\bigcup_{\substack{d_{1}, \ldots, d_{n} \in\{0, \ldots, q-1\} \\ d_{1}+\ldots+d_{n}=t}}\left\{x_{1}^{d_{1}} \ldots x_{n}^{d_{n}}\right\} .
$$

Это условие означает, что среди координатных функций отображения $f$ присутствуют все мономы степени $t$.

ЗАМЕчАНИЕ 5. Класс случайных включений $F(x) \in F\left(x^{0}\right)+B$, где $F(x)=A_{1} x+A_{2} f(x)$, а множество $R(f)=\left\{f_{1}(x), \ldots, f_{m}(x)\right\}$ удовлетворяет условию следствия 1 , содержит «заведомо совместные» системы полиномиальных уравнений (1.4). Для этих систем уравнений в качестве элементов множества (1.13) можно взять функции $f_{s}(x)=x_{t(s-1)+1} \ldots x_{t s}, \quad s=1, \ldots, l$, где $2 \leq t<n-$ целое число и $l=[n / t]-$ целая часть числа $n / t$. Тогда $\| d_{s}||=t, s=1, \ldots, l$, и условие (1.16) принимает вид

$$
\left(1-q^{-2 t}\right)^{[n / t]} \rightarrow 0 .
$$

Очевидно, это соотношение выполнено при $n \rightarrow \infty$ и заданном числе $t$.

ЗАМЕЧАНИЕ 6. В теореме работы [8] приведены условия появления предельного распределения Пуассона для числа решений $x \neq x^{0}, x^{0} \in$ $\in V^{n} \backslash\left\{0^{n}\right\}$, системы булевых уравнений $F_{t}(x)=F_{t}\left(x^{0}\right), t=1, \ldots, T$, где

$$
F_{t}(x)=\sum_{k=1}^{g_{t}} \sum_{1 \leq j_{1}<\ldots<j_{k} \leq n} a_{j_{1}, \ldots, j_{k}}^{(t)} x_{j_{1}} \ldots x_{j_{k}}
$$

и величины $g_{t}$ зависят от номеров $t=1, \ldots, T$. По сравнению со следствием 1 теорема работы [8] содержит более широкие условия для параметpa (1.8), но ограничивает вес $\left\|x^{0}\right\|$ векторов $x^{0}=x^{0}(n)$ (не допускаются векторы, имеющие при $n \rightarrow \infty$ «малый» вес, в частности, не допускается вектор 
$\left.x^{0}=0^{n}\right)$. В связи с этим отметим, что в утверждении следствия 1 сходимость к предельному распределению выполняется равномерно относительно векторов $x^{0} \in V^{n}$.

В теоремах 1 и 1а дан общий вид предельного распределения случайной величины $\xi(D, F, B)$, называемого сложным распределением Пуассона. Приведем некоторые варианты этого распределения, определяемые структурой множества $R(f)=\left\{f_{1}(x), \ldots, f_{m}(x)\right\}$.

Пусть $t_{1}, \ldots, t_{k}$ - натуральные числа. Обозначим $G\left(t_{1}, \ldots, t_{k}\right)$ подгруппу мультипликативной группы поля $K$, состоящую из всех элементов, имеющих порядки, делящие НОД $\left(t_{1}-1, \ldots, t_{k}-1\right)$.

Следствие 2. Пусть $F(x)=A_{1} x+A_{2} f(x)$ и кроме этого:

1) множество $R(f)$ определено формулой

$$
R(f)=Q_{s}^{\left(t_{0}\right)} \cup Q_{n}^{\left(t_{1}\right)} \cup \ldots \cup Q_{n}^{\left(t_{k}\right)},
$$

где $s, t_{0}, t_{1}, \ldots, t_{k}-$ заданные натуральные числа, $1 \leq s<n, 1 \leq t_{0} \leq s(q-1)$, $2 \leq t_{1}<\ldots<t_{k}<n(q-1)$

2) $a B=B$ при всех $a \in G\left(t_{1}, \ldots, t_{k}\right)$;

3) выполнены условия $n, T \rightarrow \infty, T \Delta \rightarrow 0$ и соотномение

$$
q^{n-T}|B| \rightarrow \lambda, \quad 0<\lambda<\infty .
$$

Тогда:

1) если $G\left(t_{0}, t_{1}, \ldots, t_{k}\right)<G\left(t_{1}, \ldots, t_{k}\right)$, то распределение случайной величины $\xi\left(V^{n} \backslash\left\{0^{n}\right\}, F, B\right)$ сходится к распределению случайной величины $g \pi_{g}\left(\lambda_{g}\right)+h \pi_{h}\left(\lambda_{h}\right)$, где

$$
\begin{gathered}
g=\left|G\left(t_{0}, t_{1}, \ldots, t_{k}\right)\right|, \quad h=\left|G\left(t_{1}, \ldots, t_{k}\right)\right|, \\
\lambda_{g}=\lambda\left(1-p\left(s, t_{0}\right)\right) / g, \\
\lambda_{h}=\lambda p\left(s, t_{0}\right) / h, \\
p\left(s, t_{0}\right)=\sum_{0 \leq i<t_{0} /(q-1)} C_{s}^{i}((q-1) / q)^{i}(1 / q)^{s-i} ;
\end{gathered}
$$

2) если $G\left(t_{0}, t_{1}, \ldots, t_{k}\right)=G\left(t_{1}, \ldots, t_{k}\right)$, то распределение случайной величинь

$$
\xi\left(V^{n} \backslash\left\{0^{n}\right\}, F, B\right) /\left|G\left(t_{1}, \ldots, t_{k}\right)\right|
$$

сходится к распределению Пуассона с параметром $\lambda /\left|G\left(t_{1}, \ldots, t_{k}\right)\right|$. 
ЗАМЕЧАНИЕ 7. Условию 2 следствия 2 удовлетворяют, в частности, множества $\left\{b \in V^{T}:\|b\| \leq r\right\}$ и $\left\{b \in V^{T}:\|b\|=r\right\}$ при любом $0 \leq r \leq n$, поскольку для них $a B=B, \forall a \in K \backslash\{0\}$.

Следствие 3. Пусть $F(x)=A_{1} x+A_{2} f(x)$, множество $R(f)$ определено по формуле

$$
R(f)=Q_{n}^{\left(t_{1}\right)} \cup \ldots \cup Q_{n}^{\left(t_{k}\right)},
$$

где $t_{1}, \ldots, t_{k}-$ заданные натуральные числа, причем $2 \leq t_{1}<\ldots<t_{k}<n(q-$ - 1), выполнены условия 2 и 3 следствия 2. Тогда распределение случайной величинь

$$
\xi\left(V^{n} \backslash\left\{0^{n}\right\}, F, B\right) /\left|G\left(t_{1}, \ldots, t_{k}\right)\right|
$$

сходится к распределению Пуассона с параметром $\lambda /\left|G\left(t_{1}, \ldots, t_{k}\right)\right|$.

Отметим, что в следствиях 2 и 3 степени мономов $x_{1}^{d_{1}} \ldots x_{n}^{d_{n}}$ в разложениях координатных функций $F_{i}(x), i=1, \ldots, T$ фиксированы. При использовании моделей случайных включений более естественными представляются условия следствия 4.

Следствие 4. Пусть $F(x)=A_{1} x+A_{2} f(x)$ и кроме этого:

1) выполнено, по крайней мере, одно из двух включений:

$$
\begin{gathered}
R(f) \supseteq Q_{n}^{(2)}, \\
R(f) \supseteq Q_{n}^{\left(t_{1}\right)} \cup Q_{n}^{\left(t_{2}\right)},
\end{gathered}
$$

где $2<t_{1}<t_{2}$, НОД $\left(t_{1}-1, t_{2}-1\right)=1$;

2) выполнены условия $n, T \rightarrow \infty, T \Delta \rightarrow 0$,

$$
q^{n-T}|B| \rightarrow \lambda, \quad 0<\lambda<\infty .
$$

Тогда:

1) распределение случайной величины $\xi\left(V^{n} \backslash\left\{0^{n}\right\}, F, B\right)$ сходится $\kappa$ распределению Пуассона с параметром $\lambda$;

2) если выполнено условие (B), то распределение случайной величины $\xi\left(V^{n} \backslash\left\{x^{0}\right\}, F, B \mid x^{0}\right)$ сходится к распределению Пуассона с параметром $\lambda$ равномерно относительно векторов $x^{0} \in V^{n}$.

ЗАМЕчАНИЕ 8. Класс случайных включений $A_{1} x+A_{2} f(x) \in B$, для которых выполнено условие 1 следствия 4, содержит системы полиномиальных уравнений (1.4). 
Условие $T \Delta \rightarrow 0$ в теоремах 1 и 1а означает асимптотическую равномерность распределений (1.7) элементов матрицы $A=\left(A_{1}, A_{2}\right)$. Приведем теперь главный результат настоящей работы - предельную теорему Пуассона, в которой допускается асимптотическая неравномерность и, более того, вырождение распределений элементов $A$.

Пусть

$$
\bar{Q}_{n}^{(g)}=\bigcup_{t=2}^{g} Q_{n}^{(t)}=\bigcup_{t=2}^{g} \bigcup_{\substack{d_{1}, \ldots,+d_{n} \in\{0, \ldots, q-1\} \\ d_{1}+\ldots+d_{n}=t}}\left\{x_{1}^{d_{1}} \ldots x_{n}^{d_{n}}\right\} .
$$

Для натуральных чисел $r$ и $g$ положим

$$
l(r, g)=\sum_{i=1}^{\min (r, g)} C_{r}^{i}
$$

Далее предполагаем, что числа $r=r(n), g=g(n)$ зависят от параметра $n$.

Tеорема 3. Пусть $K=G F(2), F(x)=A_{1} x+A_{2} f(x), R(f)=\bar{Q}_{n}^{(g)}$, $D \subseteq V^{n} \backslash\left\{0^{n}\right\}$, выполнены условия $n, T \rightarrow \infty, r, g \rightarrow \infty,|D| \rightarrow \infty$, и соотношения

$$
\begin{gathered}
\rho\left(D_{f}\right) \rho(B) \rightarrow 0, \\
2^{-T}|D||B| \rightarrow \lambda, \quad 0<\lambda<\infty, \\
\frac{(1+\Delta)^{T}|\{x \in D:|| x||<r\}|}{|D|} \rightarrow 0, \\
T \Delta^{l(r-j, g-j)} \rightarrow 0, \quad j=0,1, \ldots
\end{gathered}
$$

Тогда распределение случайной величины $\xi(D, F, B)$ сходится к распределению Пуассона с параметром $\lambda$.

Следствие 5. Пусть $K=G F(2), F(x)=A_{1} x+A_{2} f(x), R(f)=\bar{Q}_{n}^{(g)}$, выполнены условия $n, T \rightarrow \infty$ и соотномения $\log _{2} n \leq g \leq n$,

$$
\begin{gathered}
2^{n-T}|B| \rightarrow \lambda, \quad 0<\lambda<\infty, \\
|B| \leq 2^{\delta T}, \\
0 \leq \Delta \leq\left(2\left(1-n^{-1} \psi(n)\right)\right)^{1-\delta}-1,
\end{gathered}
$$


где $0 \leq \delta=\delta(n)<1,0<\psi(n)=o(n) u$

$$
\frac{\ln (1-\delta(n))}{\psi(n)} \rightarrow 0, \quad \frac{\ln ^{2} n}{\psi(n)} \rightarrow 0, \quad n \rightarrow \infty .
$$

Тогда распределение случайной величины $\xi\left(V^{n} \backslash\left\{0^{n}\right\}, F, B\right)$ сходится к распределению Пуассона с параметром $\lambda$.

ЗАМЕЧАНИЕ 9. Пусть в условиях (1.27), (1.28) величина $\delta(n)$ стремится к нулю. Тогда условия следствия 5 допускают возможность асимптотического вырождения распределений элементов матрицы $A$ (см. (1.7)).

Доказательство теоремы 3 приведено в разделе 2. Раздел 3 посвящен доказательству теоремы 2 и следствий $2-5$.

\section{2. Доказательство теоремы 3}

Пусть $I\{E\}$ обозначает индикатор случайного события $E$. Тогда

$$
\xi(D, F, B)=\sum_{(x, b) \in J} I\{F(x)=b\}
$$

где $J=D \times B$. Воспользуемся известной теоремой Б. А. Севастьянова о предельном распределении Пуассона суммы зависимых индикаторов (см. $[5,6])$. Условия этой теоремы используют понятие исключительных множеств. Для их построения в нашем случае потребуется ряд определений.

Пусть $D(r)=\{x \in D:\|x\| \geq r\}$. Введем множества

$$
D_{k, j}^{f}(r)=\left\{\left(x^{1}, \ldots, x^{k}\right) \in D^{k}(r): \operatorname{rank}\left(\left(x^{1}, f\left(x^{1}\right)\right), \ldots,\left(x^{k}, f\left(x^{k}\right)\right)\right)=j\right\}
$$

и $D_{k}^{f}(r)=\bigcup_{j=1}^{k-1} D_{k, j}^{f}(r)$. Отметим, что

$$
\left|D_{k, j}^{f}(r)\right| \leq \sum_{s=1}^{j}\left|\left\{\left(x^{1}, \ldots, x^{k}\right) \in D^{k}: \operatorname{rank}\left(x^{1}, \ldots, x^{k}\right)=s\right\}\right| .
$$

Поэтому

$$
\left|D_{k, j}^{f}(r)\right| \leq \sum_{s=1}^{j} C_{k}^{s} q^{s(k-s)}|D|^{s} .
$$

Положим $J(r)=D(r) \times B$ и

$$
J_{k}(r)=\left\{\left(\left(x^{1}, b^{1}\right), \ldots,\left(x^{k}, b^{k}\right) \in J^{k}(r):\left(x^{\alpha}, b^{\alpha}\right) \neq\left(x^{\beta}, b^{\beta}\right)(\alpha \neq \beta)\right\} .\right.
$$


Определим исключительные множества $I_{k}(r) \subset J_{k}(r)$ равенством

$$
I_{k}(r)=\left\{\left(\left(x^{1}, b^{1}\right), \ldots,\left(x^{k}, b^{k}\right)\right) \in J_{k}(r):\left(x^{1}, \ldots, x^{k}\right) \in D_{k}^{f}(r)\right\} .
$$

Согласно теореме Б. А. Севастьянова, утверждение теоремы 3 будет доказано, если показать, что

$$
\begin{gathered}
\sum_{(x, b) \in J \backslash J(r)} \mathbf{P}\{F(x)=b\} \rightarrow 0, \\
\sum_{(x, b) \in J(r)} \mathbf{P}\{F(x)=b\} \rightarrow \lambda, \\
\max _{(x, b) \in J(r)} \mathbf{P}\{F(x)=b\} \rightarrow 0,
\end{gathered}
$$

и при всех $k=2,3, \ldots$ (используем обозначение $\left.v^{i}=\left(x^{i}, b^{i}\right)\right)$

$$
\begin{gathered}
\max _{\left(v^{1}, \ldots, v^{k}\right) \in J_{k}(r) \backslash I_{k}(r)}\left|\frac{\mathbf{P}\left\{F\left(x^{1}\right)=b^{1}, \ldots, F\left(x^{k}\right)=b^{k}\right\}}{\mathbf{P}\left\{F\left(x^{1}\right)=b^{1}\right\} \cdot \ldots \cdot \mathbf{P}\left\{F\left(x^{k}\right)=b^{k}\right\}}-1\right| \rightarrow 0, \\
\sum_{\left(v^{1}, \ldots, v^{k}\right) \in I_{k}(r)} \prod_{j=1}^{k} \mathbf{P}\left\{F\left(x^{j}\right)=b^{j}\right\} \rightarrow 0, \\
\sum_{\left(v^{1}, \ldots, v^{k}\right) \in I_{k}(r)} \mathbf{P}\left\{F\left(x^{1}\right)=b^{1}, \ldots, F\left(x^{k}\right)=b^{k}\right\} \rightarrow 0 .
\end{gathered}
$$

Перед проверкой условий (2.2)-(2.7) приведем вспомогательное утверждение. Пусть множества $\bar{Q}_{n}^{(g)}$ определяются формулами $(1.20)$, а числа $l_{j}(r, g)=l(r-$ $-j+1, g-j+1), j=1,2, \ldots$ (см. (1.21)).

Лемма 1. Пусть $K=G F(2), F(x)=A_{1} x+A_{2} f(x), R(f)=\bar{Q}_{n}^{(g)}$, $\left(x^{1}, \ldots, x^{k}\right) \in D_{k, j}^{f}(r), 1 \leq j \leq \min (k, r, g), b^{1}, \ldots, b^{k} \in V^{T}$. Тогда при всех $k=1,2, \ldots$

$$
\mathbf{P}\left\{F\left(x^{1}\right)=b^{1}, \ldots, F\left(x^{k}\right)=b^{k}\right\} \leq\left(\frac{1+\Delta^{l_{j}(r, g)}}{2}\right)^{j T}
$$

если $j \leq k-1$, а если $j=k$, то

$$
\left(\frac{1-\Delta^{l_{k}(r, g)}}{2}\right)^{k T} \leq \mathbf{P}\left\{F\left(x^{1}\right)=b^{1}, \ldots, F\left(x^{k}\right)=b^{k}\right\} \leq\left(\frac{1+\Delta^{l_{k}(r, g)}}{2}\right)^{k T}
$$


Доказательство. Соотношение $F\left(x^{S}\right)=b^{S}$ представляет собой систему $A y=b^{s}$ из $T$ линейных уравнений со случайной матрицей $A=\left(A_{1}, A_{2}\right)$ относительно вектора $y=\left(x^{s}, f\left(x^{s}\right)\right)$. Пусть вектор $y$ имеет не менее $l$ ненулевых координат и при этом $y_{v_{1}}=\ldots=y_{v_{l}}=1$ для некоторых номеров $v_{1}, \ldots, v_{l} \in\left\{v \in \overline{1, n+m}: y_{v}=1\right\}$. Тогда, используя равенства $\Delta_{i, v}(k)=2 \mathbf{P}\left\{a_{i, v}=k\right\}-1$ и $\sum_{k \in K} \Delta_{i, v}(k)=0$, получаем

$$
\begin{gathered}
\mathbf{P}\left\{a_{i, v_{1}} y_{v_{1}}+\ldots+a_{i, v_{l}} y_{v_{l}}=c\right\}=\sum \prod_{w=1}^{l} \mathbf{P}\left\{a_{i, v_{w}}=c_{w}\right\}= \\
=\frac{1}{2}+\frac{1}{2^{l}} \sum \Delta_{i, v_{1}}\left(c_{1}\right) \cdot \ldots \cdot \Delta_{i, v_{l}}\left(c_{l}\right),
\end{gathered}
$$

где $c \in K$, а оба суммирования проводятся по всем $c_{1}, \ldots, c_{l} \in K$, для которых выполнено равенство $c_{1}+\ldots+c_{l}=c$. Значит,

$$
\frac{1-\Delta^{l}}{2} \leq \mathbf{P}\left\{a_{i, v_{1}} y_{v_{1}}+\ldots+a_{i, v_{l}} y_{v_{l}}=c\right\} \leq \frac{1+\Delta^{l}}{2} .
$$

Следовательно,

$$
\left(\frac{1-\Delta^{l}}{2}\right)^{T} \leq \mathbf{P}\left\{F\left(x^{s}\right)=b^{s} \mid E\right\} \leq\left(\frac{1+\Delta^{l}}{2}\right)^{T},
$$

где

$$
F\left(x^{s}\right)=A y, \quad y=\left(x^{s}, f\left(x^{s}\right)\right),
$$

и $E$ - любое событие, которое не зависит от совокупности случайных элементов, стоящих в столбцах матрицы $A$ с номерами $v_{1}, \ldots, v_{l} \in\{v \in$ $\left.\in \overline{1, n+m}: y_{v}=1\right\}$.

Воспользуемся этими соображениями для того, чтобы доказать неравенства (2.8) и (2.9). Пусть $\left(x^{1}, \ldots, x^{k}\right) \in D_{k, 1}^{f}(r)$. Из равенства $R(f)=\bar{Q}_{n}^{(g)}$ следует, что вектор $\left(x^{1}, f\left(x^{1}\right)\right)$ имеет не менее $\sum_{i=1}^{\min (r, g)} C_{r}^{i}=l_{1}(r, g)$ ненулевых координат. Поэтому согласно (2.10)

$$
\left(\frac{1-\Delta^{l_{1}(r, g)}}{2}\right)^{T} \leq \mathbf{P}\left\{F\left(x^{1}\right)=b^{1}\right\} \leq\left(\frac{1+\Delta^{l_{1}(r, g)}}{2}\right)^{T},
$$

при этом

$$
\mathbf{P}\left\{F\left(x^{1}\right)=b^{1}, \ldots, F\left(x^{k}\right)=b^{k}\right\} \leq \mathbf{P}\left\{F\left(x^{1}\right)=b^{1}\right\} \leq\left(\frac{1+\Delta^{l_{1}(r, g)}}{2}\right)^{T} .
$$

Значит, в случае, когда $k \geq j=1$, соотношения (2.8), (2.9) выполнены. 

положим

Пусть $\left(x^{1}, \ldots, x^{k}\right) \in D_{k, j}^{f}(r), k \geq j \geq 2$. Без ограничения общности

$$
\begin{gathered}
\operatorname{rank}\left(\left(x^{1}, f\left(x^{1}\right)\right), \ldots,\left(x^{j}, f\left(x^{j}\right)\right)\right)=j, \\
r \leq\left\|x^{1}\right\| \leq\left\|x^{2}\right\| \leq \ldots \leq\left\|x^{j}\right\| .
\end{gathered}
$$

Для $2 \leq u \leq j$ через $\eta_{u}(f)$ обозначим число номеров таких координат $f_{i}\left(x^{u}\right)$ вектора $f\left(x^{u}\right)$, для которых выполнены равенства

$$
f_{i}\left(x^{u}\right)=1, \quad f_{i}\left(x^{1}\right)=\ldots=f_{i}\left(x^{u-1}\right)=0 .
$$

Пусть $r_{u}=\left\|x^{u}\right\|$. Выберем в матрице, составленной из строк $x^{1}, \ldots, x^{u}$, подматрицу $M_{u}$ размера $u \times r_{u}$, образованную $r_{u}$ столбцами с ненулевыми элементами вектора $x^{u}$. Не ограничивая общности, будем полагать, что эти столбцы имеют номера $1, \ldots, r_{u}$. Из условий $(2.12),(2.13)$ и того, что все векторы $x^{1}, \ldots, x^{u}$ различны, следует, что каждая строка матрицы $M_{u}$ с номером $s=1, \ldots, u-1$ содержит нулевой элемент. Следовательно, для некоторого $1 \leq v \leq u-1$ найдутся такие номера $1 \leq \omega_{1}<\ldots<\omega_{v} \leq r_{u}$, что $x_{\omega_{1}}^{u} \ldots x_{\omega_{v}}^{u}=1$ и $x_{\omega_{1}}^{s} \ldots x_{\omega_{v}}^{s}=0$ при всех $s=1, \ldots, u-1$. Очевидно, что при любом наборе номеров $1 \leq k_{1}<\ldots<k_{t} \leq r_{u}, t \geq v$, включающем номера $\omega_{1}, \ldots, \omega_{v}$, также выполнены равенства $x_{k_{1}}^{u} \ldots x_{k_{t}}^{u}=1, x_{k_{1}}^{s} \ldots x_{k_{t}}^{s}=0$ при всех $s=1, \ldots, u-1$. Из этих наборов нас интересуют лишь наборы не более чем по $g$ номеров. Число таких наборов равно

$$
\sum_{i=0}^{\min \left\{g-v, r_{u}-v\right\}} C_{r_{u}-v}^{i} \geq \sum_{i=0}^{\min \{g-u+1, r-u+1\}} C_{r-u+1}^{i}=l_{u}(r, g) .
$$

Здесь были использованы неравенства $r_{u} \geq r$ и $v \leq u-1$. Из (2.14), равенства $R(f)=Q_{n}^{(g)}$ и наших определений следует, что

$$
\eta_{u}(f) \geq l_{u}(r, g), \quad u=2, \ldots, j .
$$

Из (2.15) и (2.10) вытекает, что при всех $u=2, \ldots, j$

$$
\begin{gathered}
\left(\frac{1-\Delta^{l_{u}(r, g)}}{2}\right)^{T} \leq \mathbf{P}\left\{F\left(x^{u}\right)=b^{u} \mid F\left(x^{1}\right)=b^{1}, \ldots, F\left(x^{u-1}\right)=b^{u-1}\right\} \leq \\
\leq\left(\frac{1+\Delta^{l_{u}(r, g)}}{2}\right)^{T} .
\end{gathered}
$$

Осталось воспользоваться соотношениями

$$
\mathbf{P}\left\{F\left(x^{1}\right)=b^{1}, \ldots, F\left(x^{k}\right)=b^{k}\right\} \leq \mathbf{P}\left\{F\left(x^{1}\right)=b^{1}, \ldots, F\left(x^{j}\right)=b^{j}\right\},
$$




$$
\begin{aligned}
& \quad \mathbf{P}\left\{F\left(x^{1}\right)=b^{1}, \ldots, F\left(x^{j}\right)=b^{j}\right\}=\mathbf{P}\left\{F\left(x^{1}\right)=b^{1}\right\} \times \\
& \times \prod_{u=2}^{j} \mathbf{P}\left\{F\left(x^{u}\right)=b^{u} \mid F\left(x^{1}\right)=b^{1}, \ldots, F\left(x^{u-1}\right)=b^{u-1}\right\}
\end{aligned}
$$

и оценками (2.11), (2.16). Лемма 2.1 доказана.

Приступим к проверке соотношений (2.2)-(2.7). Проверим соотношение (2.2). Учитывая, что для любого $x \in V^{n} \backslash\left\{0^{n}\right\}$ выполнено неравенство $\mathbf{P}\{F(x)=b\} \leq((1+\Delta) / 2)^{T}$, получаем

$$
\begin{gathered}
\sum_{(x, b) \in J \backslash J(r)} \mathbf{P}\{F(x)=b\} \leq|B||\{x \in D:\|x\|<r\}|\left(\frac{1+\Delta}{2}\right)^{T}= \\
=2^{-T}|B||D| \times \frac{|\{x \in D:\|x\|<r\}|(1+\Delta)^{T}}{|D|} .
\end{gathered}
$$

Согласно условиям (1.23), (1.24) правая часть в цепочке соотношений (2.17) стремится к нулю. Значит, соотношение (2.2) выполнено.

Из неравенств (2.8), (2.9), условий (1.23), (1.25) и (2.2) следуют соотношения (2.3) и (2.4).

Перед проверкой соотношений (2.5)-(2.7) отметим, что она вполне аналогична проверке подобных соотношений в доказательстве теоремы 4 работы [1]. Проверим условие (2.5). Согласно определениям множество $J_{k}(r) \backslash I_{k}(r)$ образуют все наборы $\left(\left(x^{1}, b^{1}\right), \ldots,\left(x^{k}, b^{k}\right)\right)$, в которых $\left(x^{1}, \ldots, x^{k}\right) \in D_{k, k}^{f}(r)$. Из неравенств (2.9) вытекает, что для них

$$
\left(\frac{1-\Delta^{l_{k}(r, g)}}{1+\Delta^{l_{k}(r, g)}}\right)^{k T} \leq \frac{\mathbf{P}\left\{F\left(x^{1}\right)=b^{1}, \ldots, F\left(x^{k}\right)=b^{k}\right\}}{\mathbf{P}\left\{F\left(x^{1}\right)=b^{1}\right\} \cdot \ldots \cdot \mathbf{P}\left\{F\left(x^{k}\right)=b^{k}\right\}} \leq\left(\frac{1+\Delta^{l_{k}(r, g)}}{1-\Delta^{l_{k}(r, g)}}\right)^{k T} .
$$

Поэтому из условия (1.25) следует (2.5).

Проверим соотношение (2.6) при $k \geq 2$. Используя неравенства (2.9) (где $k=1)$ и (2.1), получаем

$$
\begin{gathered}
\sum_{\left(v^{1}, \ldots, v^{k}\right) \in I_{k}(r)} \prod_{s=1}^{k} \mathbf{P}\left\{F\left(x^{s}\right)=b^{s}\right\} \leq \\
\leq \sum_{\left(x^{1}, \ldots, x^{k}\right) \in D_{k}^{f}(r)} \prod_{s=1}^{k} \mathbf{P}\left\{F\left(x^{s}\right) \in B\right\}=\sum_{j=1}^{k-1} \sum_{\left(x^{1}, \ldots, x^{k}\right) \in D_{k, j}^{f}(r)} \prod_{s=1}^{k} \mathbf{P}\left\{F\left(x^{s}\right) \in B\right\} \leq
\end{gathered}
$$




$$
\begin{gathered}
\leq \sum_{j=1}^{k-1}\left|D_{k, j}^{f}(r)\right|\left(\frac{1+\Delta^{l_{1}(r, g)}}{2}\right)^{k T}|B|^{k} \leq \\
\leq\left(\frac{|D| \cdot|B|}{2^{T}}\right)^{k}\left(1+\Delta^{l_{1}(r, g)}\right)^{k T} \frac{1}{|D|^{k}} \sum_{j=1}^{k-1} \sum_{s=1}^{j} C_{k}^{s} q^{s(k-s)}|D|^{s} .
\end{gathered}
$$

Из условий (1.23), (1.25) и условия $|D| \rightarrow \infty$ следует, что выражение в правой части цепочки соотношений (2.18) стремится к нулю при всех $k \geq 2$. Соотношение (2.6) доказано.

Наконец, проверим соотношение (2.7). Опять $k \geq 2$. Из определений следует равенство

$$
\begin{aligned}
& \sum_{\left(v^{1}, \ldots, v^{k}\right) \in I_{k}(r)} \mathbf{P}\left\{F\left(x^{1}\right)=b^{1}, \ldots, F\left(x^{k}\right)=b^{k}\right\}= \\
= & \sum_{\substack{\left(v^{1}, \ldots, v^{k}\right) \in J_{k}(r) \\
\left(x^{1}, \ldots, x^{k}\right) \in D_{k}^{f}(r)}} \mathbf{P}\left\{F\left(x^{1}\right)=b^{1}, \ldots, F\left(x^{k}\right)=b^{k}\right\} .
\end{aligned}
$$

Введем в рассмотрение множества

$$
\bar{D}_{k, j}^{f}(r)=D_{k, j}^{f}(r) \cap\left\{\left(x^{1}, \ldots, x^{k}\right) \in(D(r))^{k}: x^{\alpha} \neq x^{\beta}(\alpha \neq \beta)\right\} .
$$

Заметим, что $\bar{D}_{k, 1}^{f}(r)=\varnothing, k=2,3, \ldots$. Положим $\bar{D}_{k}^{f}(r)=\bigcup_{j=2}^{k-1} \bar{D}_{k, j}^{f}(r)$.

Лемма 2. Пусть $F(x)=A_{1} x+A_{2} f(x),\left(\left(x^{1}, b^{1}\right), \ldots,\left(x^{k}, b^{k}\right)\right) \in J_{k}(r)$, $\left(x^{1}, \ldots, x^{k}\right) \in D_{k}^{f}(r) \backslash \bar{D}_{k}^{f}(r)$. Тогда

$$
\mathbf{P}\left\{F\left(x^{1}\right)=b^{1}, \ldots, F\left(x^{k}\right)=b^{k}\right\}=0 .
$$

Утверждение леммы вытекает из определений множеств $J_{k}(r)$ и $\bar{D}_{k}^{f}(r)$. Из (2.19) и леммы 2 следует, что

$$
\begin{aligned}
& \sum_{\left(v^{1}, \ldots, v^{k}\right) \in I_{k}(r)} \mathbf{P}\left\{F\left(x^{1}\right)=b^{1}, \ldots, F\left(x^{k}\right)=b^{k}\right\}= \\
= & \sum_{\substack{\left(v^{1}, \ldots, v^{k}\right) \in J_{k}(r) \\
\left(x^{1}, \ldots, x^{k}\right) \in \bar{D}_{k}^{f}(r)}} \mathbf{P}\left\{F\left(x^{1}\right)=b^{1}, \ldots, F\left(x^{k}\right)=b^{k}\right\} .
\end{aligned}
$$


Теперь рассмотрим произвольный набор векторов $x^{1}, \ldots, x^{k} \in V^{n}$. Ему соответствует система $L_{f}\left(x^{1}, \ldots, x^{k}\right)$ однородных линейных уравнений над полем $K=G F(2)$ вида

$$
c_{1}\left(x^{1}, f\left(x^{1}\right)\right)+\ldots+c_{k}\left(x^{k}, f\left(x^{k}\right)\right)=0
$$

(возможно пустая), состоящая из всех линейных соотношений, которым удовлетворяет набор векторов $\left(x^{1}, f\left(x^{1}\right)\right), \ldots,\left(x^{k}, f\left(x^{k}\right)\right)$. Системе уравнений $L_{f}\left(x^{1}, \ldots, x^{k}\right)$ сопоставим идентичную по составу систему $L_{b}\left(x^{1}, \ldots, x^{k}\right)$ из линейных уравнений относительно $b^{1}, \ldots, b^{k} \in B$. Обозначим через $B\left(x^{1}, \ldots, x^{k}\right)$ множество решений $\left(b^{1}, \ldots, b^{k}\right) \in B^{k}$ системы $L_{b}\left(x^{1}, \ldots, x^{k}\right)$.

Лемма 3. Пусть $\left(b^{1}, \ldots, b^{k}\right) \notin B\left(x^{1}, \ldots, x^{k}\right),\left(x^{1}, \ldots, x^{k}\right) \in \bar{D}_{k}^{f}(r)$. Тогда

$$
\mathbf{P}\left\{F\left(x^{1}\right)=b^{1}, \ldots, F\left(x^{k}\right)=b^{k}\right\}=0 .
$$

Доказательство леммы 3 повторяет доказательство леммы 4.3 работы [1], и мы его не приводим.

Из леммы 3 следует, что

$$
\begin{gathered}
\sum_{\substack{\left(v^{1}, \ldots, v^{k}\right) \in J_{k}(r) \\
\left(x^{1}, \ldots, x^{k}\right) \in \bar{D}_{k}(r)}} \mathbf{P}\left\{F\left(x^{1}\right)=b^{1}, \ldots, F\left(x^{k}\right)=b^{k}\right\}= \\
=\sum_{j=2}^{k-1} \sum_{\substack{\left.\left(x^{1}, \ldots, x^{k}\right) \in \bar{D}_{k, j}(r) \\
b^{1}, \ldots, b^{k}\right) \in B\left(x^{1}, \ldots, x^{k}\right)}} \mathbf{P}\left\{F\left(x^{1}\right)=b^{1}, \ldots, F\left(x^{k}\right)=b^{k}\right\} .
\end{gathered}
$$

Здесь нам понадобится оценка мощности множества $B\left(x^{1}, \ldots, x^{k}\right)$.

Лемма 4. Пусть $F(x)=A_{1} x+A_{2} f(x), 2 \leq j \leq k-1,\left(x^{1}, \ldots, x^{k}\right) \in$ $\in \bar{D}_{k, j}^{f}(r)$. Тогдa

$$
\left|B\left(x^{1}, \ldots, x^{k}\right)\right| \leq \rho(B)|B|^{j}
$$

Лемма 4 повторяет (с некоторыми отличиями в обозначениях) лемму 4.4 работы [1]. Поэтому ее доказательство мы не приводим. 
Применим лемму 4 и оценку (2.8) к выражению в правой части цепочки неравенств (2.21), а результат подставим в (2.20). Получим

$$
\begin{gathered}
\sum_{\left(v^{1}, \ldots, v^{k}\right) \in I_{k}(r)} \mathbf{P}\left\{F\left(x^{1}\right)=b^{1}, \ldots, F\left(x^{k}\right)=b^{k}\right\} \leq \\
\leq \sum_{j=2}^{k-1} \sum_{\left(x^{1}, \ldots, x^{k}\right) \in \bar{D}_{k, j}^{f}(r)} \sum_{\left(b^{1}, \ldots, b^{k}\right) \in B\left(x^{1}, \ldots, x^{k}\right)}\left(\frac{1+\Delta^{l_{j}(r, g)}}{2}\right)^{j T} \leq \\
\leq \sum_{j=2}^{k-1} \sum_{\left(x^{1}, \ldots, x^{k}\right) \in \bar{D}_{k, j}^{f}(r)}\left|B\left(x^{1}, \ldots, x^{k}\right)\right|\left(\frac{1+\Delta^{l_{j}(r, g)}}{2}\right)^{j T} \leq \\
\leq \rho(B) \sum_{j=2}^{k-1} \sum_{\left(x^{1}, \ldots, x^{k}\right) \in \bar{D}_{r, j}^{f}(r)}|B|^{j}\left(\frac{1+\Delta^{l_{j}(r, g)}}{2}\right)^{j T} \leq \\
\leq \rho(B) \sum_{j=2}^{k-1}\left|\bar{D}_{k, j}^{f}(r)\right| \cdot|B|^{j}\left(\frac{1+\Delta^{l_{j}(r, g)}}{2}\right)^{j T} \leq \\
\leq \rho(B) \sum_{j=2}^{k-1} \frac{\left|\bar{D}_{k, j}^{f}\right|}{|D|^{j}} \cdot \sum_{j=2}^{k-1}\left(\frac{\left(1+\Delta^{l_{j}(r, g)}\right)^{T}|D| \cdot|B|}{2^{T}}\right)^{j},
\end{gathered}
$$

где

$$
\begin{gathered}
\bar{D}_{k, j}^{f}=\left\{\left(x^{1}, \ldots, x^{k}\right) \in D^{k}: \operatorname{rank}\left(\left(x^{1}, f\left(x^{1}\right)\right), \ldots,\left(x^{k}, f\left(x^{k}\right)\right)\right)=j,\right. \\
\left.x^{\alpha} \neq x^{\beta}(\alpha \neq \beta)\right\} .
\end{gathered}
$$

Лемма 5. Пусть $2 \leq j \leq k-1, n \rightarrow \infty$, а множество $D$ меняется так, что $\rho\left(D_{f}\right) \rightarrow 0$. Тогда

$$
\left|\bar{D}_{k, j}^{f}\right| \cdot|D|^{-j} \rightarrow 0 .
$$

Доказательство леммы 5 повторяет доказательство леммы 4.5 работы [1], и мы его также не приводим.

Из условий (1.22), (1.23), (1.25) и леммы 5 следует, что выражение в правой части цепочки неравенств (2.22) стремится к нулю. Значит, условие (2.7) тоже выполнено. Теорема 3 доказана. 


\section{3. Доказательства теоремы 2 и следствий 2-5}

В пунктах 3.1-3.3 рассматривается случай $K=G F(q)$.

\section{1. Доказательство теоремы 2}

Нам понадобится следующий результат.

Лемма 6. При всех $x^{0} \in V^{n}$ выполнено равенство

$$
\rho\left(\left(V^{n}\right)_{f^{\left(x^{0}\right)}}\right)=\rho\left(\left(V^{n}\right)_{f}\right) .
$$

Доказательство леммы. Напомним, что

$$
\begin{gathered}
\left(V^{n}\right)_{f}=\left\{(x, f(x))=\left(x_{1}, \ldots, x_{n}, f_{1}(x), \ldots, f_{m}(x)\right), x \in V^{n}\right\}, \\
\left(V^{n}\right)_{f^{\left(x^{0}\right)}}=\left\{\left(x, f^{\left(x^{0}\right)}(x)\right)=\left(x_{1}, \ldots, x_{n}, f_{1}^{\left(x^{0}\right)}(x), \ldots, f_{m}^{\left(x^{0}\right)}(x)\right), x \in V^{n}\right\},
\end{gathered}
$$

где

$$
f_{s}^{\left(x^{0}\right)}(x)=f_{s}\left(x+x^{0}\right)-f_{s}\left(x^{0}\right), \quad s=1, \ldots, m .
$$

Согласно определению, приведенному в разделе 1 ,

$$
\rho\left(\left(V^{n}\right)_{f}\right)=\frac{N_{f}}{q^{2 n}}, \quad \rho\left(\left(V^{n}\right)_{f^{\left(x^{0}\right)}}\right)=\frac{N_{f\left(x^{0}\right)}}{q^{2 n}},
$$

где $N_{f}$ и $N_{f\left(x^{0}\right)}$ - максимально возможное при $a_{1}, a_{2}, a_{3} \in K \backslash\{0\}, c=$ $=\left(c^{1}, c^{2}\right) \in V^{n+m}$ число решений уравнения

$$
a_{1} y^{1}+a_{2} y^{2}+a_{3} y^{3}=c
$$

относительно тройки векторов $\left(y^{1}, y^{2}, y^{3}\right) \in\left(\left(V^{n}\right)_{f}\right)^{3}$ в первом случае и относительно тройки векторов $\left(y^{1}, y^{2}, y^{3}\right) \in\left(\left(V^{n}\right)_{f\left(x^{0}\right)}\right)^{3}$ во втором случае.

В первом случае уравнение (3.2) относительно переменных $x_{i}^{1}, x_{i}^{2}, x_{i}^{3}$, $i=1, \ldots, n$, перепишется как система из $n+m$ уравнений (назовем ее системой (3.3)):

$$
\begin{gathered}
a_{1} x_{i}^{1}+a_{2} x_{i}^{2}+a_{3} x_{i}^{3}=c_{i}^{1}, \quad i=1, \ldots, n, \\
a_{1} f_{s}\left(x^{1}\right)+a_{2} f_{s}\left(x^{2}\right)+a_{3} f_{s}\left(x^{3}\right)=c_{s}^{2}, \quad s=1, \ldots, m .
\end{gathered}
$$


Во втором случае уравнение (3.2) относительно переменных $x_{i}^{1}, x_{i}^{2}, x_{i}^{3}, i=$ $=1, \ldots, n$ перепишется как система из $n+m$ уравнений (назовем ее системой (3.4)):

$$
\begin{gathered}
a_{1} x_{i}^{1}+a_{2} x_{i}^{2}+a_{3} x_{i}^{3}=c_{i}^{1}, \quad i=1, \ldots, n, \\
a_{1} f_{s}\left(x^{1}+x^{0}\right)+a_{2} f_{s}\left(x^{2}+x^{0}\right)+a_{3} f_{s}\left(x^{3}+x^{0}\right)= \\
=c_{s}^{2}+\left(a_{1}+a_{2}+a_{3}\right) f_{s}\left(x^{0}\right), \quad s=1, \ldots, m .
\end{gathered}
$$

Система (3.4) приводится к виду (3.3) заменой правых частей и линейной заменой переменных. Поэтому $N_{f}=N_{f\left(x^{0}\right)}$ и выполнено равенство (3.1). Лемма 6 доказана.

Перейдем к доказательству теоремы 2. Не нарушая общности рассуждений, будем рассматривать мономы $x_{1}^{d_{1}^{s}} \ldots x_{n}^{d_{n}^{s}}$ из множества $Q\left(d^{1}, \ldots, d^{l}\right)$ в качестве первых $l$ координатных функций $f_{1}(x), \ldots, f_{l}(x)$ отображения $f(x)=\left(f_{1}(x), \ldots, f_{m}(x)\right)$. Напомним, что $m=m(n) \geq l=\left|Q\left(d^{1}, \ldots, d^{l}\right)\right|$.

Исключим из системы (3.3) последние $m-l$ уравнений подсистемы (3.3.2), оставив в ней лишь уравнения, соответствующие мономам из множества $Q\left(d^{1}, \ldots, d^{l}\right)$. Обозначим полученную систему как $\left(3.3^{*}\right)$, а максимальное число ее решений - как $N_{f}^{*}$. Очевидно, что

$$
N_{f}^{*} \geq N_{f} .
$$

Система $\left(3.3^{*}\right)$ является системой из $n+l$ уравнений относительно $3 n$ неизвестных элементов $x_{i}^{1}, x_{i}^{2}, x_{i}^{3}, i=1, \ldots, n$, поля $K$. Нетрудно убедиться, что ее уравнения можно распределить по $l+1$ системам следующим образом. Обозначим через $I_{s}$ множество существенных переменных монома $f_{s}(x)=$ $=f_{s}\left(x_{1}, \ldots, x_{n}\right)$. Согласно определениям $\left|I_{s}\right|=\left\|d^{s}\right\|$. Система с номером $s=$ $=1, \ldots, l$ имеет вид

$$
\begin{gathered}
a_{1} f_{s}\left(x^{1}\right)+a_{2} f_{s}\left(x^{2}\right)+a_{3} f_{s}\left(x^{3}\right)=c_{s}^{2}, \\
a_{1} x_{i}^{1}+a_{2} x_{i}^{2}+a_{3} x_{i}^{3}=c_{i}^{1}, \quad i \in I_{s} .
\end{gathered}
$$

К системе (3.6. $(l+1))$ отнесем все остальные $n-\left\|d^{1}\right\|-\ldots-\left\|d^{l}\right\|$ уравнений подсистемы (3.3.1).

Обозначим через $N_{f}^{(s)}$ максимальное (по всем допустимым вариантам выбора коэффициентов и правых частей уравнений) число решений системы (3.6.s). Так как множества неизвестных, входящих в системы (3.6.s), $s=1, \ldots, l+1$, попарно не пересекаются, то

$$
N_{f}^{*}=\prod_{s=1}^{l+1} N_{f}^{(s)} .
$$


Ниже мы покажем, что

$$
\begin{gathered}
N_{f}^{(s)} \leq q^{2\left\|d^{s}\right\|}-1, \quad s=1, \ldots, l, \\
N_{f}^{(l+1)}=q^{2\left(n-\left\|d^{1}\right\|-\ldots-\left\|d^{l}\right\|\right)} .
\end{gathered}
$$

Из (3.7)-(3.9) и (3.5) следуют неравенства

$$
N_{f} \leq N_{f}^{*} \leq q^{2 n} \prod_{s=1}^{l}\left(1-q^{-2\left\|d^{s}\right\|}\right) .
$$

Поэтому с учетом (3.1) получаем:

$$
\rho\left(\left(V^{n}\right)_{f^{\left(x^{0}\right)}}\right)=\rho\left(\left(V^{n}\right)_{f}\right) \leq \prod_{s=1}^{l}\left(1-q^{-2\left\|d^{s}\right\|}\right) .
$$

Осталось воспользоваться тем, что для некоторого $C>0$

$$
\left|\rho\left(\left(V^{n} \backslash\left\{0^{n}\right\}\right)_{f^{\left(x^{0}\right)}}\right)-\rho\left(\left(V^{n}\right)_{f^{\left(x^{0}\right)}}\right)\right| \leq \frac{C}{q^{n}} .
$$

Для завершения доказательства теоремы 2 надо убедиться в справедливости соотношений (3.8) и (3.9). Равенство (3.9) почти очевидно. Система (3.6. $(l+1))$ позволяет выразить элементы $x_{i}^{3}$ через $x_{i}^{1}$ и $x_{i}^{2}$ для всех $i \in\{1, \ldots, n\} \backslash\left(I_{1} \cup \ldots \cup I_{l}\right)$, что и приводит к равенству (3.9).

Докажем неравенство (3.8). Рассмотрим систему (3.6.s), $1 \leq s \leq l$. Используя линейные уравнения, выразим неизвестные $x_{i}^{3}$ через $x_{i}^{1}$ и $x_{i}^{2}$ (полагаем $a_{3}=1$ ) и подставим их в нелинейное уравнение. Получим уравнение

$$
a_{1} f_{s}\left(x^{1}\right)+a_{2} f_{s}\left(x^{2}\right)+f_{s}\left(c^{1}-a_{1} x^{1}-a_{2} x^{2}\right)=c_{s}^{2},
$$

где $f_{s}(x)=x_{j_{1}} \ldots x_{j_{k}}$ при некоторых $2 \leq k \leq n$ и $1 \leq j_{1}<\ldots<j_{k} \leq n$. Другими словами,

$$
a_{1} \prod_{u=1}^{k} x_{j_{u}}^{1}+a_{2} \prod_{u=1}^{k} x_{j_{u}}^{2}+\prod_{u=1}^{k}\left(c_{j_{u}}^{1}-a_{1} x_{j_{u}}^{1}-a_{2} x_{j_{u}}^{2}\right)=c_{s}^{2} .
$$

Уравнение (3.11) является уравнением от $2\left\|d^{s}\right\|$ неизвестных из поля $K$. Покажем, что при любом задании элементов $a_{1} \neq 0, a_{2} \neq 0, c_{s}^{2}$ и вектора $c^{1}$ левая часть уравнения (3.11) не равна тождественно константе; тогда число решений этого уравнения меньше $q^{2\left\|d^{s}\right\|}$. 
Выражение в левой части (3.11) является многочленом степени $k \geq 2$. Опустив в нем члены степени меньше $k$, получим выражение

$$
\begin{gathered}
a_{1} \prod_{u=1}^{k} x_{j_{u}}^{1}+a_{2} \prod_{u=1}^{k} x_{j_{u}}^{2}+(-1)^{k} \prod_{u=1}^{k}\left(a_{1} x_{j_{u}}^{1}+a_{2} x_{j_{u}}^{2}\right)= \\
=\left(a_{1}+\left(-a_{1}\right)^{k}\right) \prod_{u=1}^{k} x_{j_{u}}^{1}+\left(a_{2}+\left(-a_{1}\right)^{k}\right) \prod_{u=1}^{k} x_{j_{u}}^{2}+ \\
+(-1)^{k} \sum_{\substack{U \subset\{1, \ldots, k\} \\
1 \leq|U| \leq k-1}} \prod_{u \in U}\left(a_{1} x_{j_{u}}^{1}\right) \prod_{u \in\{1, \ldots, k\} \backslash U}\left(a_{2} x_{j_{u}}^{2}\right) .
\end{gathered}
$$

Очевидно, что все мономы, входящие в сумму по множествам $U \subset\{1, \ldots, k\}$, $1 \leq|U| \leq k-1$, имеют коэффициенты, отличные от нуля, и не могут сократиться при приведении подобных членов. Значит, левая часть уравнения (3.11) не является тождественной константой. Следовательно, выполнено неравенство (3.8). Доказательство теоремы 2 завершено.

\section{2. Доказательство следствия 2}

Выделим в множестве $D_{f}$ подмножества (здесь $\left.x^{(s)}=\left(x_{1}, \ldots, x_{s}\right)\right)$

$$
\begin{aligned}
& M_{f}^{(0)}=\left\{y=(x, f(x)) \in D_{f}:\left\|x^{(s)}\right\|<t_{0} /(q-1),\|x\| \geq t_{k}\right\}, \\
& M_{f}^{(1)}=\left\{y=(x, f(x)) \in D_{f}:\left\|x^{(s)}\right\| \geq t_{0} /(q-1),\|x\| \geq t_{k}\right\} .
\end{aligned}
$$

Нетрудно убедиться, что векторы $y^{1}=\left(x^{1}, f\left(x^{1}\right)\right), y^{2}=\left(x^{2}, f\left(x^{2}\right)\right) \in$ $\in M_{f}^{(0)}$ являются подобными в том и только том случае, когда $x^{2}=$ $=a x^{1}$, причем $a \in G\left(t_{1}, \ldots, t_{k}\right)$. Следовательно, множество $M_{f}^{(0)}$ разбивается на $\left|M_{f}^{(0)}\right| /\left|G\left(t_{1}, \ldots, t_{k}\right)\right|$ классов подобных векторов, каждый мощности $\left|G\left(t_{1}, \ldots, t_{k}\right)\right|$. В силу условия $a B=B, a \in G\left(t_{1}, \ldots, t_{k}\right)$, множество $M_{f}^{(0)} \times B$ разбивается на $\left|M_{f}^{(0)}\right| \cdot|B| /\left|G\left(t_{1}, \ldots, t_{k}\right)\right|$ классов такой же мощности.

Аналогичными рассуждениями показывается, что множество $M_{f}^{(1)} \times B$ разбивается на $\left|M_{f}^{(1)}\right| \cdot|B| /\left|G\left(t_{0}, t_{1}, \ldots, t_{k}\right)\right|$ классов подобных векторов, каждый мощности $\left|G\left(t_{0}, t_{1}, \ldots, t_{k}\right)\right|$.

Пусть $G\left(t_{0}, t_{1}, \ldots, t_{k}\right)<G\left(t_{1}, \ldots, t_{k}\right)$. Положим $H_{f}=\left(V^{n} \backslash\left\{0^{n}\right\}\right)_{f}$. Используя указанные выше разбиения множеств $M^{(0)} \times B, M^{(1)} \times B$, равенство 
$\left|H_{f}\right|=q^{n}-1$ и легко проверяемые соотношения

$$
\frac{\left|M^{(0)}\right|}{\left|H_{f}\right|} \rightarrow p\left(s, t_{0}\right), \quad \frac{\left|M^{(1)}\right|}{\left|H_{f}\right|} \rightarrow 1-p\left(s, t_{0}\right), \quad n \rightarrow \infty,
$$

где

$$
p\left(s, t_{0}\right)=\sum_{0 \leq i<\left(t_{0} /(q-1)\right)} C_{s}^{i}((q-1) / q)^{i}(1 / q)^{s-i},
$$

получаем две цепочки асимптотических равенств:

$$
\begin{gathered}
q^{-T} l_{\left|G\left(t_{1}, \ldots, t_{k}\right)\right|}\left(H_{f}, B\right) \approx q^{-T} \frac{\left|M^{(0)}\right| \cdot|B|}{\left|G\left(t_{1}, \ldots, t_{k}\right)\right|} \approx \frac{\lambda p\left(s, t_{0}\right)}{\left|G\left(t_{1}, \ldots, t_{k}\right)\right|}, \\
q^{-T} l_{\left|G\left(t_{0}, t_{1}, \ldots, t_{k}\right)\right|}\left(H_{f}, B\right) \approx q^{-T} \frac{\left|M^{(1)}\right| \cdot|B|}{\left|G\left(t_{0}, t_{1}, \ldots, t_{k}\right)\right|} \approx \frac{\lambda\left(1-p\left(s, t_{0}\right)\right)}{\left|G\left(t_{0}, t_{1}, \ldots, t_{k}\right)\right|}
\end{gathered}
$$

(здесь и далее запись $a(n) \approx b(n)$ означает, что $a(n)=b(n)(1+o(1))$ при $n \rightarrow$ $\rightarrow \infty)$. Вместе с этим получаем, что

$$
q^{-T} l_{r}\left(H_{f}, B\right) \leq q^{n-T}|B| \cdot \frac{\left|H_{f}\right|-\left|M^{(0)}\right|-\left|M^{(1)}\right|}{\left|H_{f}\right|}
$$

для значений

$$
r \in\{1, \ldots, q-1\}, \quad r \neq\left|G\left(t_{1}, \ldots, t_{k}\right)\right|, \quad r \neq\left|G\left(t_{0}, t_{1}, \ldots, t_{k}\right)\right| .
$$

Следовательно,

$$
q^{-T} l_{r}\left(H_{f}, B\right) \rightarrow 0
$$

для указанных в (3.16) значений параметра $r$. Из (3.13), (3.14), (3.17) следует, что условие (1.10) теоремы 1 выполнено, причем

$$
\begin{aligned}
\lambda_{\left|G\left(t_{1}, \ldots, t_{k}\right)\right|} & =\frac{\lambda p\left(s, t_{0}\right)}{\left|G\left(t_{1}, \ldots, t_{k}\right)\right|}, \\
\lambda_{\left|G\left(t_{0}, t_{1}, \ldots, t_{k}\right)\right|} & =\frac{\lambda\left(1-p\left(s, t_{0}\right)\right)}{\left|G\left(t_{0}, t_{1}, \ldots, t_{k}\right)\right|},
\end{aligned}
$$

и $\lambda_{r}=0$ для указанных в (3.16) значений параметра $r$. 
Согласно теореме 2 и замечанию 4 кроме условия (1.10) выполнено и условие (1.9) теоремы 1. Все остальные условия теоремы 1 содержатся в условиях следствия. Первое утверждение следствия 2 доказано.

Пусть $G\left(t_{0}, t_{1}, \ldots, t_{k}\right)=G\left(t_{1}, \ldots, t_{k}\right)$. Тогда вместо цепочек (3.13), (3.14) получим одну цепочку асимптотически эквивалентных величин

$$
q^{-T} l_{\left|G\left(t_{1}, \ldots, t_{k}\right)\right|}\left(H_{f}, B\right) \approx q^{-T} \frac{\left(\left|M^{(0)}\right|+\left|M^{(1)}\right|\right)|B|}{\left|G\left(t_{1}, \ldots, t_{k}\right)\right|} \approx \frac{\lambda}{\left|G\left(t_{1}, \ldots, t_{k}\right)\right|} .
$$

Используя неравенства (3.15) для

$$
r \in\{1, \ldots, q-1\}, \quad r \neq\left|G\left(t_{1}, \ldots, t_{k}\right)\right|,
$$

получим для этих значений соотношения (3.17). Таким образом, установим, что условие (1.10) теоремы 1 выполнено так, что

$$
\lambda_{\left|G\left(t_{1}, \ldots, t_{k}\right)\right|}=\frac{\lambda}{\left|G\left(t_{1}, \ldots, t_{k}\right)\right|},
$$

и $\lambda_{r}=0$ для указанных в (3.18) значений параметра $r$. Осталось заметить, что условие (1.9) теоремы 1 выполнено согласно теореме 2 и замечанию 4. Следствие 2 доказано.

\section{3. Доказательства следствий 3 и 4}

Доказательство следствия 3 и первого утверждения следствия 4 проводятся по той же схеме, что и доказательство следствия 2. Теперь вместо двух множеств $M_{f}^{0}$ и $M_{f}^{1}$ рассматривается одно множество

$$
M_{f}=\left\{y=(x, f(x)) \in D_{f}:\|x\| \geq t\right\},
$$

где $t=t_{k}$ в случае следствия 3 и $t=2$ или $t=t_{2}$ в случае следствия 4 (в зависимости от того, которое из условий (1.18) и (1.19) считается выполненным). В условиях следствия 3 векторы $y^{1}=\left(x^{1}, f\left(x^{1}\right)\right), y^{2}=\left(x^{2}, f\left(x^{2}\right)\right) \in M_{f}$ являются подобными в том и только том случае, когда $x^{2}=a x^{1}$, причем $a \in G\left(t_{1}, \ldots, t_{k}\right)$. Поэтому опять приходим к выводу о том, что множество $M_{f} \times B$ разбивается на $\left|M_{f}\right| \cdot|B| /\left|G\left(t_{1}, \ldots, t_{k}\right)\right|$ классов подобных векторов мощности $G\left(t_{1}, \ldots, t_{k}\right)$ каждый. В условиях следствия 4 векторы $y^{1}=\left(x^{1}, f\left(x^{1}\right)\right), y^{2}=\left(x^{2}, f\left(x^{2}\right)\right) \in M_{f}$ являются подобными в том и только том случае, когда $x^{2}=x^{1}$, т. е. когда они совпадают. Поэтому множество $M_{f} \times B$ не содержит подобных векторов.

Дальнейшие рассуждения для обоих следствий аналогичны заключительной части доказательства следствия 2, и мы их не приводим. Второе утверждение следствия 4 вытекает из теорем 1 и 2 и следствия 1. 


\section{4. Доказательство следствия 5}

Напомним, что в данном случае $K=G F(2), D=V^{n} \backslash\left\{0^{n}\right\}$ и выполнены условия (1.27) и (1.28):

$$
|B| \leq 2^{\delta T}, \quad 0 \leq \Delta \leq\left(2\left(1-n^{-1} \psi(n)\right)^{1-\delta}-1,\right.
$$

где $0 \leq \delta=\delta(n)<1,0<\psi(n)=o(n)$ и

$$
\frac{\ln (1-\delta(n))}{\psi(n)} \rightarrow 0, \quad \frac{\ln ^{2} n}{\psi(n)} \rightarrow 0, \quad n \rightarrow \infty
$$

Покажем, что в этих условиях для величины $\xi\left(V^{n} \backslash\left\{0^{n}\right\}, F, B\right)$ выполнены условия теоремы 3 .

Из условий (1.16) и (3.19) вытекает, что при достаточно больших $n$

$$
T \leq \frac{n-\log _{2} \lambda+1}{1-\delta}
$$

Положим $r=r(n)=\left[\log _{2} n\right]$. Тогда

$$
\left|\left\{x \in V^{n} \backslash\left\{0^{n}\right\}:\|x\|<r\right\}\right|<C_{n}^{\left[\log _{2} n\right]} \log _{2} n<n^{\log _{2} n} .
$$

Из (3.22) и условия (1.18) следует, что найдется такое $C_{1}(\lambda)>0$, что при достаточно больших $n$

$$
\begin{gathered}
T \ln (1+\Delta) \leq\left(n-\log _{2} \lambda+1\right) \ln \left(2\left(1-\frac{\psi(n)}{n}\right)\right) \leq \\
\leq\left(n-\log _{2} \lambda+1\right)\left(\ln 2-\frac{\psi(n)}{n}\right)= \\
=\ln \left(2^{n}-1\right)-\psi(n)-\left(\ln \left(1-2^{-n}\right)+\left(1-\log _{2} \lambda\right)\left(\ln 2-n^{-1} \psi(n)\right)\right) \leq \\
\leq \ln \left(2^{n}-1\right)-C_{1}(\lambda) \psi(n) .
\end{gathered}
$$

Из (3.20)-(3.23) следует, что в нашем случае выполнено условие (1.24) теоремы 3:

$$
\begin{gathered}
\frac{(1+\Delta)^{T}}{|D|}\left|\left\{x \in V^{n} \backslash\left\{0^{n}\right\}:\|x\|<r\right\}\right|<\frac{e^{T \ln (1+\Delta)}}{2^{n}-1} n^{\log _{2} n}< \\
<e^{-C_{1}(\lambda) \psi(n)} n^{\log _{2} n} \rightarrow 0, \quad n \rightarrow \infty .
\end{gathered}
$$


Проверим, что в нашем случае выполнено условие (1.25) теоремы 3. Так как у нас $g=g(n) \geq r$, то согласно определению (см. (1.21))

$$
l(r, g)=2^{\left[\log _{2} n\right]}-1, \quad l(r-j, g-j)=2^{\left[\log _{2} n\right]-j}-1 \geq n 2^{-j-1}-1 .
$$

Из (1.18), (3.21) и (3.24) следует, что при $j=0,1, \ldots$ и достаточно больших $n$

$$
T \Delta^{l(r-j, g-j)}<\frac{n-\log _{2} \lambda+1}{1-\delta(n)}\left(1-\frac{2 \psi(n)}{n}\right)^{n 2^{-j-1}-1}<\frac{C_{2}(\lambda)}{1-\delta(n)} n e^{-2^{-j} \psi(n)},
$$

где $C_{2}(\lambda)<\infty$. Из (3.25) и (3.20) следует (1.25).

Осталось отметить, что в случае $D=V^{n} \backslash\left\{0^{n}\right\}$ и $R(f)=\bar{Q}_{n}^{(g)}$ соотношение (1.22) вытекает из теоремы 2. Таким образом, можно воспользоваться теоремой 3. Следствие 5 доказано.

\section{Список литературы}

1. Копытщев В.А., Михайлов В.Г. Теоремы пуассоновского типа для числа специальных решений случайного линейного включения. - Дискретная математика, 2010, т. 22, вып. 2, с. 3-21.

2. Копытиев В.А., Михайлов В.Г. Теоремы пуассоновского типа для числа решений случайных включений. - Математические вопросы криптографии, 2010, т. 1, вып. 4, с. 63-84.

3. Копытцев В.А. О числе решений систем линейных булевых уравнений в множестве векторов, обладающих заданным числом единиц. Дискретная математика, 2002, т. 14, вып. 4, с. 87-109.

4. Копытиев В.А. О числе решений системы случайных линейных уравнений. - Дискретная математика, 2006, т. 18, вып. 1, с. 40-62.

5. Колчин В.Ф., Севастьянов Б.А., Чистяков В.П. Случайные размещения. - М.: Наука, 1976.

6. Севастьянов Б. А. Предельный закон Пуассона в схеме сумм зависимых случайных величин. - Теория вероятн. и ее применен., 1972, т. 17, вып. 4, c. $733-738$.

7. Михайлов В.Г. Предельные теоремы для числа решений системы случайных линейных уравнений, попавших в заданное множество. Дискретная математика, 2007, т. 19, вып. 1, с. 17-26.

8. Масол В.И. Теорема о предельном распределении числа ложных решений системы нелинейных случайных уравнений. - Теория вероятн. и ее примен., 1998, т. 43, вып. 1, с. 41-56. 